\title{
BONANZA DEL COBRE: IMPACTO MACROECONÓMICO Y DESAFÍOS DE POLÍTICA*
}

\author{
José De Gregorio
}

\begin{abstract}
El significativo aumento del precio del cobre ha puesto sobre la mesa un conjunto de temas de primera importancia. En este trabajo se abordan dos de ellos. El primero es preguntarse por qué la economía chilena no crece más rápido, tal como ocurrió en otras bonanzas del cobre. Hay un conjunto de factores que lo explican, pero aquí se argumenta que el principal elemento detrás de esta situación es la política macroeconómica que es estabilizadora, y eso es una muy buena noticia para los futuros ajustes. El segundo tema que se discute es el desafío de política fiscal, puesto que el elevado precio del cobre podría resultar en una expansión fiscal difícil de absorber sin mayores trastornos. Para ello se propone buscar formas en que, dentro de los marcos de la regla fiscal, se prioricen áreas de alto contenido social y de un positivo impacto macroeconómico, como es el fortalecimiento del seguro de desempleo.
\end{abstract}

José De Gregorio. Doctor en Economía, Massachusetts Institute of Technology (MIT). Vicepresidente del Banco Central de Chile. Fue Ministro de Economía, Minería y Energía.

* Preparado para el seminario Administrando el Auge del Cobre, organizado por Expansiva y Libertad y Desarrollo, 30 de mayo, 2006. Agradezco los comentarios de María Cristina Betancour, Pablo García, Eric Parrado, Rodrigo Valdés y un árbitro anónimo, así como a participantes en un seminario en el Banco Central. Las opiniones expresadas aquí son de mi exclusiva responsabilidad y no representan necesariamente al Banco ni al resto de su Consejo.

Estudios Públicos, 103 (invierno 2006). 
$\mathrm{E}$

l precio del cobre está llegando a niveles record históricos (Figura No 1). Después de haber alcanzado en los años 2000-2002 el trienio de menor precio del cobre desde la Gran Depresión, hoy día estamos puntualmente en valores superiores a los máximos promedios anuales históricos ${ }^{1}$. Si lograremos o no batir este récord dependerá de la persistencia del aumento que estamos observando, pero ciertamente, nuestras proyecciones son que en términos reales tendremos el precio promedio anual más alto de los últimos 30 años $^{2}$. Cualquier cosa puede pasar en los años que vienen, y a pesar que el precio del cobre puede seguir aumentando algo más, es razonable pensar que este debiera retroceder. El problema es que no sabemos hasta dónde puede llegar ni cuándo se devolverá y es en este escenario de incertidumbre en el cual debemos hacer nuestras políticas económicas.

Este ambiente de bonanza es una muy buena noticia para la economía chilena, y pensar lo contrario es al menos paradójico. A pesar de que podemos enfrentar posibles tensiones sectoriales, debemos aprovechar esta oportunidad para consolidar una política económica que dé estabilidad al país y por esa vía contribuir a mejorar las condiciones de vida de todos los chilenos.

En esta nota quisiera abordar dos temas. El primero es el de entender por qué nuestra economía está creciendo menos que en el pasado, cuando también se produjeron auges del precio del cobre. El segundo es el de discutir las posibles implicancias de políticas de este auge del cobre.

En mi discusión quisiera remarcar los siguientes puntos:

- La principal razón por la cual la economía chilena no está creciendo al 8 o 9\%, como en ciclos pasados de alzas de precios, es que las políticas monetaria, cambiaria y fiscal proveen de un importante elemento estabilizador del ciclo económico ${ }^{3}$. Más allá de eventuales cambios, y si los hay no son muy significativos, en el crecimiento potencial, esto se trata de un resultado muy bueno, pues garantiza

${ }^{1}$ El deflactor utilizado para el precio del cobre es el IPP de EE.UU. Se eligió éste por ser el más representativo de cambios que pudieran enfrentar los costos asociados a la producción de este commodity. Si se usara el IPC de los EE.UU. los precios pre1990 serían mayores.

${ }^{2}$ El promedio en moneda de hoy desde 1935, incluyendo la proyección del 2006, ha sido 153 US\$c/lb y de los últimos 30 años 132 US\$c/lb.

${ }^{3}$ En las expansiones del cobre anteriores el producto creció a 8\% y más y por eso se usan estas cifras. Sin embargo, aún hay un margen de crecimiento no explicado y del cual hay evidencia que pueda afirmar con algún grado de rigor de dónde proviene. Pueden ser efectos cíclicos o cambios de tendencia, aunque algo de esto se discute más adelante. 
FIGURA N ${ }^{\circ} 1: \quad$ PRECIO DEL COBRE

(cUS\$/lb; US\$ 2005)

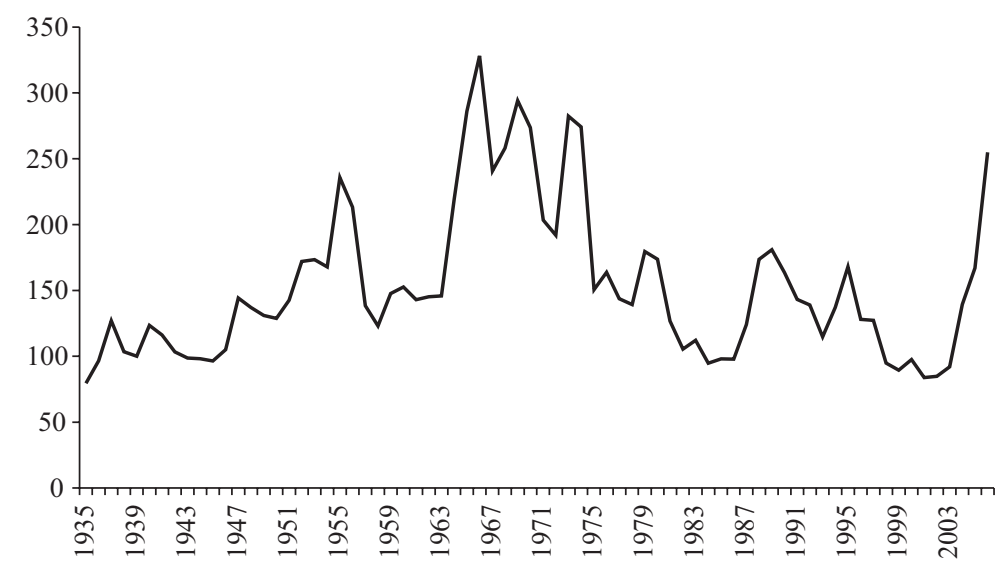

Fuente: Comisión Chilena del Cobre.

que cuando la situación externa se revierta, no sufriremos las recesiones a que estábamos acostumbrados en el pasado. Esto ya fue comprobado en los primeros años de esta década.

- La consolidación de este esquema de políticas que genera mayor estabilidad se basa también en la credibilidad que ha conseguido el manejo macroeconómico en el país. Esto aumenta la eficacia de las políticas y reduce los costos del ciclo económico.

- $\quad$ El régimen de flotación cambiaria ha sido exitoso. Ciertamente tiene costos en términos de volatilidad del peso, la que ha aumentado con respecto al pasado, pero la libre flotación ha evitado incentivar la entrada de capitales de corto plazo que podrían aumentar las presiones cambiarias y ha facilitado el ajuste de la economía a cambios en el escenario externo ${ }^{4}$.

- $\quad$ La evidencia muestra que la evolución del tipo de cambio real ha sido consistente con la enorme ganancia en términos de intercambio ocurrida en los últimos años y, dadas las condiciones actuales, es difícil pensar que el tipo de cambio real se pueda apreciar adicionalmente de manera significativa.

${ }^{4}$ La volatilidad del tipo de cambio en Chile ha aumentado recientemente, aunque esto ha ocurrido en muchos otros países cuyas monedas flotan desde hace mucho tiempo. 
- La política fiscal basada exitosamente en la regla del superávit estructural de 1\% enfrenta un desafío doble. En primer lugar ha sido diseñada para gastar los componentes permanentes del aumento de ingresos, lo cual es recomendable desde el punto de vista de la fortaleza de nuestras cuentas fiscales. Sin embargo, la fórmula de implementación puede agregar un grado de impulso fiscal con dificultades de ser absorbido sin mayor trastorno dada la actual capacidad productiva.

\section{1. ¿Por qué la economía no crece mucho más?}

Un alza de los términos de intercambio genera aumentos en el ingreso nacional que tendrían un efecto en la demanda agregada a través de, entre otros, los ingresos fiscales. Este aumento de la demanda genera expansiones del producto y cambios en precios relativos. Los efectos finales dependerán de cuán duradero sea este shock y cuál sea la reacción de política económica, la que incluso puede influir sobre la percepción de la persistencia del shock afectando las expectativas privadas.

$\mathrm{Al}$ considerar el efecto directo sobre la demanda es difícil explicar los ciclos asociados al cobre en la economía chilena. Durante mucho tiempo ha existido la inquietud de por qué aparentemente la economía sobrerreacciona al precio del cobre. En particular caídas bruscas del precio del cobre nos llevan a recesiones y aumentos acelerados a expansiones insostenibles. Los mecanismos macroeconómicos tradicionales parecieran ser incapaces de explicar por qué el consumo, y más en general, el gasto agregado, pueden crecer a tasas superiores a las que podrían explicarse por el impacto sobre el ingreso nacional que tiene un aumento del precio del cobre. En otras palabras, pareciera que en el pasado aumentos del precio del cobre habrían gatillado una euforia difícil de explicar. El cobre es una importante fuente de recursos fiscales, pero su importancia no es tan grande como para explicar sus efectos sobre la economía agregada. El sector cuprífero representó en 2005 el 13\% del PIB en términos nominales. Aproximadamente dos tercios de ese porcentaje corresponden a empresas extranjeras y su impacto en el empleo es menor.

Si bien aquí no pretendo resumir toda la evidencia, en la Figura $\mathrm{N}^{\circ} 2$ se ve que con precios incluso no tan altos como los de hoy, la economía crecía más rápido. Es así como en los años 1978-1980 crecimos a 8,1\%, en el período 1988-1989 lo hicimos a 8,9\%, y en el 95, a 10,1\%. Sin duda que hay muchas otras razones que nos ayudan a explicar el crecimiento efectivo de cada año, pero estas cifras ilustran claramente que hoy día estamos crecien- 
FIGURA N ${ }^{\circ}$ 2: $\quad$ PIB - PRECIO COBRE

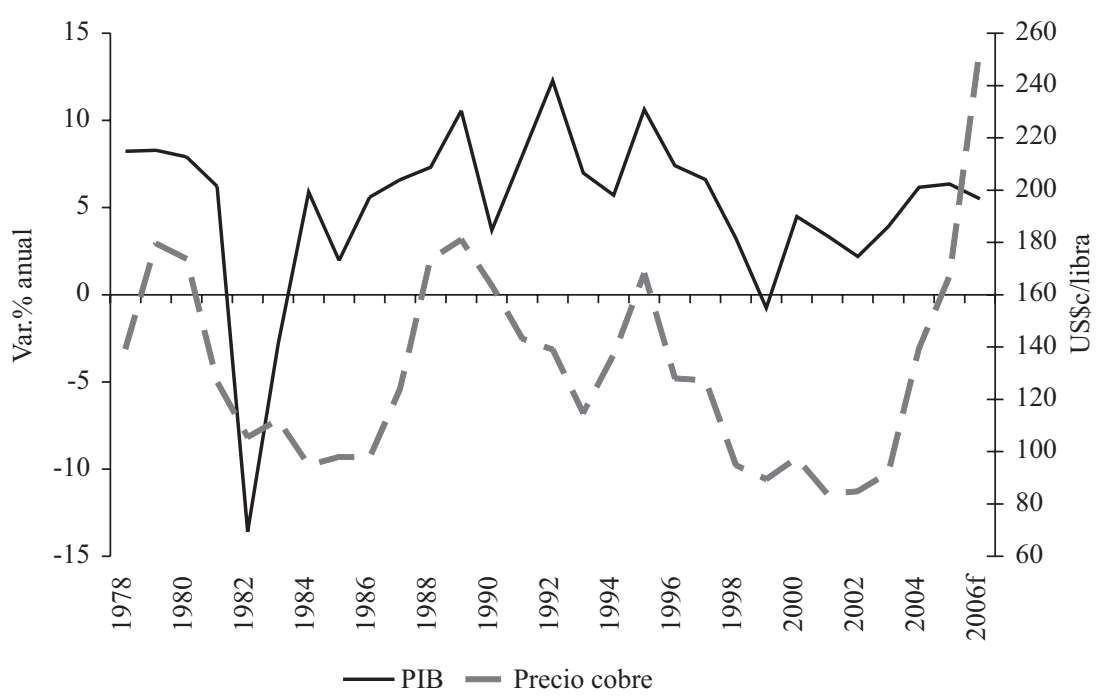

Fuente: Comisión Chilena del Cobre, Banco Central de Chile.

do menos que otros períodos de bonanza cuprífera ${ }^{5}$. Y es esta la inquietud que quiero abordar.

Una primera explicación para el elevado impacto que históricamente ha tenido el precio del cobre sobre la economía nacional es que cuando el cobre sube, porque la economía mundial está vigorosa, o el dólar se deprecia en el mundo, todos los commodities suben de precio. De hecho, la evolución del precio del cobre está muy correlacionada con los términos del intercambio, más allá del efecto directo del precio del cobre (Figura No 3). En consecuencia, podemos pensar que el precio del cobre elevado es un reflejo de muchas otras condiciones internacionales positivas. No obstante, en este último ciclo se observa una caída y posterior alza más moderadas en los términos de intercambio que en el precio del cobre ${ }^{6}$. Sin embargo, esto

${ }^{5}$ Mirando la evidencia histórica se observa que el año récord fue 1966 con un precio promedio anual en moneda de hoy de 3,3 dólares la libra. Ese mismo año la economía chilena creció $11,2 \%$, tres veces más que en los tres años previos y que en los tres años subsecuentes.

${ }^{6}$ A partir de fines de los 90 las series de términos de intercambio y precio del cobre se separan porque el precio de las importaciones experimenta una disminución y posteriormente un aumento que compensa en parte las variaciones en los precios de las exportaciones, en particular, las de cobre. 
FIGURA N ${ }^{\circ}$ 3: TÉRMINOS DE INTERCAMBIO - PRECIO COBRE

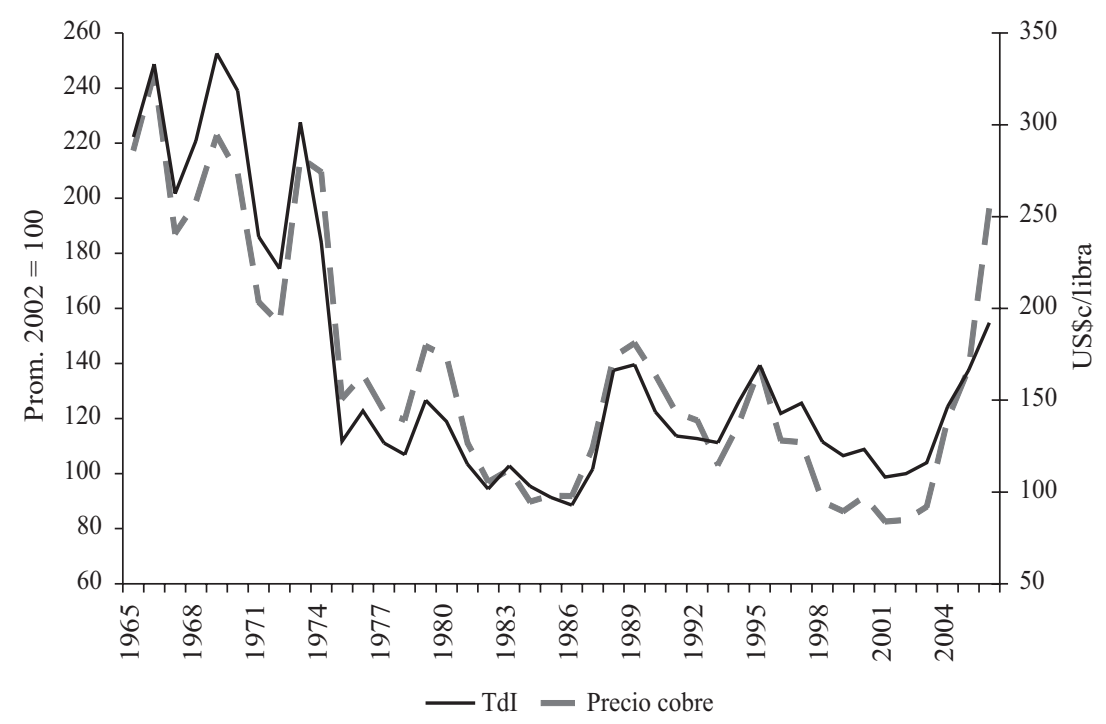

Fuente: Bennett y Valdés (2001) empalmando con datos de valor unitario de exportaciones e importaciones del Banco Central de Chile, y Comisión Chilena del Cobre.

tampoco pareciera ser suficiente para explicar la sensibilidad que en el pasado tenía el PIB a fluctuaciones del precio del cobre, ni tampoco su contraste con la situación actual, pues los términos de intercambio han estado aumentando significativamente el 2004-2006. Es por ello que es necesario explorar con más detenimiento canales de transmisión adicionales ${ }^{7}$.

A continuación revisaré las dos causas principales que se han dado en la discusión económica sobre la sobrerreacción de la economía a cambios en el precio del cobre:

- Cambios drásticos en las condiciones financieras externas.

- Políticas macroeconómicas procíclicas.

\subsection{Cambios en las condiciones financieras externas}

A partir de la crisis asiática quedó claro que los mercados financieros pueden castigar severamente a las economías emergentes, muchas ve-

${ }^{7}$ De hecho, Franken et al. (2005) encuentran que son los términos de intercambio y no el precio del cobre los que afectan al producto. 
ces sin mayor fundamento. Por otra parte existen períodos en los cuales el apetito por invertir en las economías emergentes aumenta a niveles que para ellas son difíciles de absorber. Las economías emergentes pueden contagiarse de turbulencias en mercados distantes y muchas veces desconectados.

Si se revisa la evolución de los mercados financieros durante los noventa se observa un período de expansión acelerada en la primera mitad que culmina con un dramático colapso del financiamiento después de la crisis asiática. Estos son los conocidos sudden stops (Calvo, 2005). En el caso de la economía chilena se ha argumentado que cuando el precio del cobre aumenta, el valor del colateral de la economía aumenta, esto reduce restricciones de liquidez en los mercados financieros y el gasto se desborda. En el caso opuesto, y que se ha argumentado podría haber ocurrido con la recesión del 99 en Chile, es que cuando el precio del cobre cae se corta el financiamiento externo para Chile pues el valor de su colateral, cobre, cae (Caballero, 2002). Esto genera fluctuaciones del tipo del acelerador financiero discutido para mercados de capitales domésticos, extendidas a mercados globales (Bernanke et al., 1996).

Si bien este mecanismo puede haber operado en algún momento, es difícil pensar que fue relevante en la economía chilena durante los noventa. En primer lugar, Chile no perdió acceso a los mercados financieros internacionales durante la segunda mitad de los noventa. Más aún, después de la crisis asiática en Chile no se detuvieron las entradas de capitales, como ocurre en todos los países que sufren del sudden stop, sino que la reversión de la cuenta de capitales y corriente ocurre por la vía de una masiva salida de capitales, en particular fondos de pensiones y otros inversionistas que prefirieron protegerse ante una inminente depreciación del peso ${ }^{8}$. Desde el punto de vista agregado esto era consistente con una economía que se enfrentaba a una fuerte contracción del gasto que resultó en una reducción del déficit en cuenta corriente desde niveles cercanos al 5\% en 1998 a 0\% en 1999.

Es necesario aclarar que el que se argumente que no ha habido un efecto significativo desde el punto de vista de los cambios en las condiciones financieras internacionales que enfrenta Chile no significa que no exista un acelerador financiero interno. La evolución del endeudamiento, en particular de empresas de menor tamaño, después de la crisis de liquidez del 98,

\footnotetext{
${ }^{8}$ Para más detalles véase Cowan y De Gregorio (2005), donde se llama a este fenómeno un sudden start de outlflows más que un sudden stop de inflows.
} 
indica que su posición patrimonial es un importante determinante de sus capacidades de financiamiento ${ }^{9}$.

Por último, la experiencia de principios de la década actual es una primera prueba de que a pesar de tener un precio del cobre muy bajo (el trienio de menor precio desde la Gran Depresión es el 2001-2003) y una recesión internacional de por medio, la economía no colapsó, pues logró crecer a un promedio de $3,4 \%{ }^{10}$. En consecuencia, si bien el comportamiento de los mercados financieros internacionales sí es relevante para nuestro desempeño económico, está lejos de ser el principal determinante de la reacción de la economía chilena a los cambios del precio del cobre y las condiciones internacionales en general. Para entender efectivamente lo que pasa hay que mirar dentro, en particular nuestras políticas económicas.

\subsection{El marco de política macroeconómica}

Una razón tradicional por la que las economías de América Latina fluctúan excesivamente al ritmo de la economía mundial y tienen ciclos más pronunciados, es el del efecto desestabilizador de la política fiscal. Es bien sabido que la mayoría de los países de la región, con la excepción de Chile, tienen políticas fiscales procíclicas. Esto ocurre por la combinación de un mejoramiento en las condiciones de financiamiento externo y la fragilidad fiscal. Cuando la economía mundial está boyante, el apetito por riesgo de los inversionistas internacionales aumenta, lo que incentiva flujos de capitales hacia las economías emergentes. Esto permite a los gobiernos realizar gastos que antes no podían hacer por falta de financiamiento. En la raíz de este problema está la fragilidad fiscal, que impide un acceso menos restringido a los mercados internacionales. Éste no es el caso de Chile.

En el caso de Chile, de existir una política fiscal procíclica como en el resto de América Latina, con una alta probabilidad tendría su origen en movimientos del precio del cobre. Sin embargo, no es posible justificar que los aumentos del precio del cobre en los últimos 25 años hayan generado expansiones fiscales responsables de un crecimiento excesivo y causantes de nuestras recesiones. La Figura $N^{\circ} 4$ muestra que efectivamente el saldo

${ }^{9}$ Esto además ha sido confirmado en estudios de inversión donde el flujo de caja es un importante determinante de la inversión. Véase por ejemplo Medina y Valdés (1998).

${ }^{10}$ En rigor, hay que considerar que algo que evitó además una caída es que la economía ya se encontraba saliendo de una recesión, y este mal escenario internacional ayuda a explicar la reducción del crecimiento después de la incipiente recuperación del 2000. 


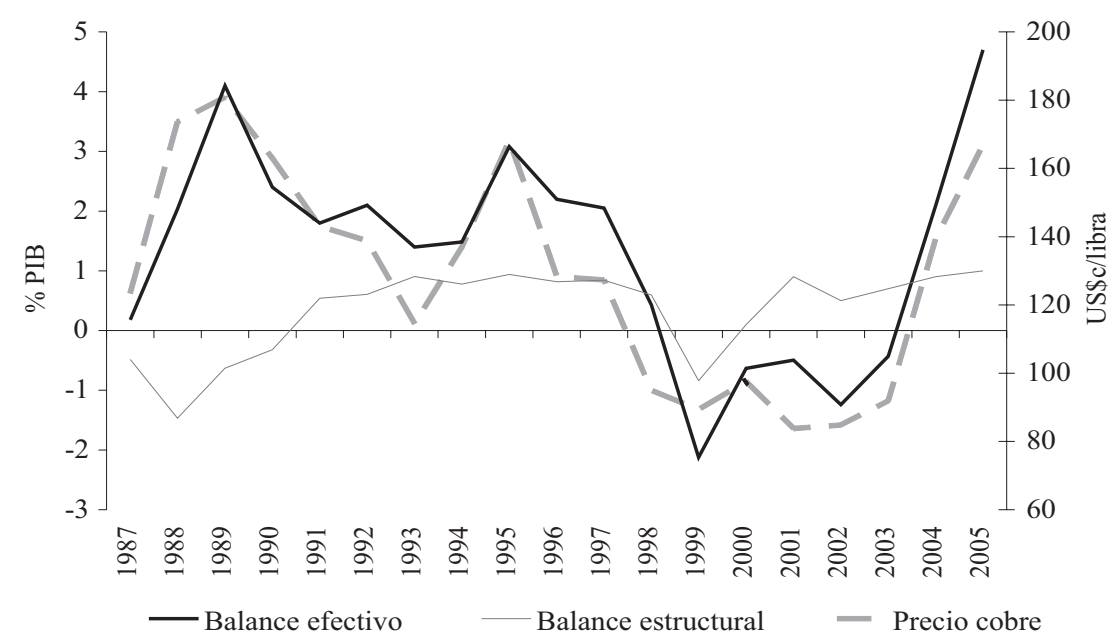

Fuente: Comisión Chilena del Cobre, Ministerio de Hacienda.

fiscal mejora cuando el precio del cobre aumenta. El precio del cobre y el balance efectivo del fisco están altamente correlacionados. Podrá discutirse en el margen acerca de su comportamiento alrededor de nuestras recesiones, pero ciertamente está muy lejos de haber sido un elemento desestabilizador. Por el contrario, mirado en una perspectiva de mediano plazo, la política fiscal chilena de los últimos 25 años ha sido estabilizadora. Esto es particularmente importante hoy, cuando el aumento del precio del cobre es muy elevado y todo el incremento se está ahorrando para dar cumplimiento a la política de mantener un superávit estructural de 1\%.

En todo caso es necesario destacar que durante los 90, con la excepción de la parte final, la política fiscal puede haber sido tan estabilizadora como la actual. Esto se observa claramente al ver que hubo un superávit estructural bastante estable y cercano a 1\% durante dicho período. Esto reafirma el hecho que es difícil pensar que la política fiscal explica, en particular comparado con el ciclo de mediados de los 90, las expansiones del PIB y gasto que ocurrieron en los 90 con la bonanza del cobre. La mayor parte de la década pasada la regla fiscal era que el gasto corriente del gobierno creciera menos que el gasto agregado. Esto tiene un elemento procíclico, el que no se captura adecuadamente en la medición del superávit estructural, aunque en todo caso es menor a juzgar por las cifras de balance fiscal. Por 
lo tanto se puede afirmar que la regla fiscal es un elemento que ha contribuido a la estabilidad del ciclo, incluso durante los 90, y por lo tanto no da cuenta del efecto estabilizador que observamos en la actualidad comparado con la menor estabilidad del pasado más reciente.

Entonces, si no ha sido la política fiscal la principal causa de los ciclos del cobre del pasado, entonces ¿por qué se inducen aumentos del gasto privado exagerados? Una primera opción es simplemente pensar que el sector privado se comporta extrañamente, pero como argumentaré aquí no me parece una respuesta razonable. A mi juicio, lo que está detrás de los desbordes de gasto privado tiene su origen en las políticas monetaria y, principalmente, la cambiaria. Aunque su influencia es algo más sutil que la de la política fiscal, su rol no es menos importante.

Un caso emblemático es el de la crisis del 82. Las cuentas fiscales estaban muy ordenadas, y el déficit en cuenta corriente en 1981 fue de la friolera suma de $13 \%$ del PIB. La economía creció a $6 \%$ con un cobre a sólo 127 centavos de dólar la libra en moneda de hoy (Figura No 5). El problema estuvo en el ámbito cambiario-financiero.

Es útil entonces repasar los efectos estabilizadores de nuestro actual esquema de políticas monetaria y cambiaria, que es conocido como metas de inflación.

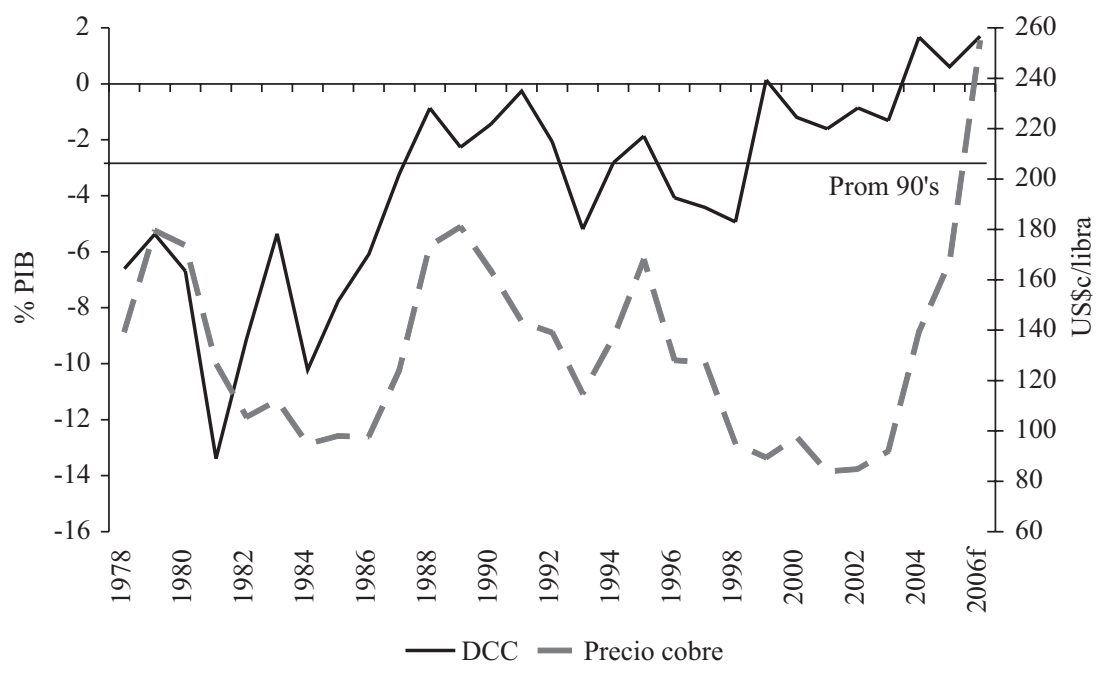

Fuente: Comisión Chilena del Cobre, Banco Central de Chile. 
En primer lugar, un esquema de metas de inflación creíble garantiza la adecuada reacción de la política monetaria a desviaciones significativas del producto de su nivel de pleno empleo. En particular, shocks positivos de demanda, que puedan provenir del mejoramiento de las condiciones externas, debieran ser acompañados de un aumento de la tasa de política monetaria que eviten un desanclaje de la inflación y permitan a la economía estabilizarse sin sufrir expansiones insostenibles y muy costosas de revertir. Si estos shocks provocan una caída del tipo de cambio que afecta las perspectivas inflacionarias, la reacción de política monetaria es atenuada. Asimismo, cuando la demanda está deprimida y la brecha del producto es negativa, las presiones deflacionarias que esto induce hacen que las tasas de interés bajen. Eso ocurrió entre el 2002 y principios del 2004 cuando la tasa de interés en Chile alcanzó sus menores niveles históricos, lo que explica de manera importante la recuperación de la economía, ya consolidada desde el año pasado.

En segundo lugar, otra pieza fundamental de este esquema es la flotación cambiaria. El tipo de cambio contribuye a la estabilidad permitiendo ajustes del tipo de cambio real a las condiciones económicas domésticas. Un tipo de cambio flexible como el que tenemos en Chile facilita el ajuste mientras vaya combinado de un esquema de política macroeconómica coherente. Si las políticas fiscal y monetaria fueran inadecuadas, sería posible tener fluctuaciones del tipo de cambio que exacerben más las fluctuaciones macroeconómicas. Cuando la situación externa es boyante, la apreciación del tipo de cambio permite quitar presión sobre la demanda agregada, la actividad e inflación, reduciendo la necesidad de efectuar movimientos bruscos en la tasa de interés. Los movimientos del tipo de cambio son un mecanismo de ajuste de la economía, el que cuando se impide que funcione tiende a generar crisis cambiarias y problemas mayores que los que se intenta resolver. Asimismo, este esquema permite que las desviaciones de su tendencia de largo plazo sean acotadas. Y tal como se ha definido la política de flotación, si éste en algún momento se desvía a rangos que atenten contra la estabilidad generando desbalances riesgosos, la opción de intervención no es descartable.

¿Qué estaría pasando por ejemplo si el Banco Central hubiera decidido, por la vía de la intervención, graduar la apreciación del peso que ha ocurrido en el último tiempo? La expectativa de que el tipo de cambio se apreciará baja el costo de endeudarse en el exterior, con lo cual habría incentivos a la entrada de capitales, se experimentarían más presiones al tipo de cambio y habría mayor crecimiento y mayor demanda. Las presiones inflacionarias inducirían un aumento de la tasa de interés con mayores im- 
pactos cambiarios. La economía crecería más, el tipo de cambio real se apreciaría, el déficit en la cuenta corriente aumentaría, el precio de las acciones y de los activos en general también aumentaría, habría euforia, y por supuesto, la reversión de esto sería más costosa. De hecho, la evidencia muestra que durante el período de masivas entradas de capitales en los noventa los países que experimentaron mayores influjos de capitales fueron aquellos que tenían un régimen cambiario administrado (Cowan y De Gregorio, 2005), independiente de si tenían o no controles de capital. Es decir, el intento de frenar las apreciaciones genera más presiones cambiarias por los incentivos a la entrada de capitales.

Al respecto, nuestra experiencia pasada es aleccionadora. En 1997, con un déficit en cuenta corriente de 4,4\% del PIB y un cobre en torno a un 1 dólar la libra, el tipo de cambio llegó a su mínimo de la década de los noventa ${ }^{11}$ (Figura $\mathrm{N}^{\mathrm{o}}$ 6). En esa época se trató de evitar la apreciación del peso. Había controles de capitales, el tipo de cambio se manejaba dentro de

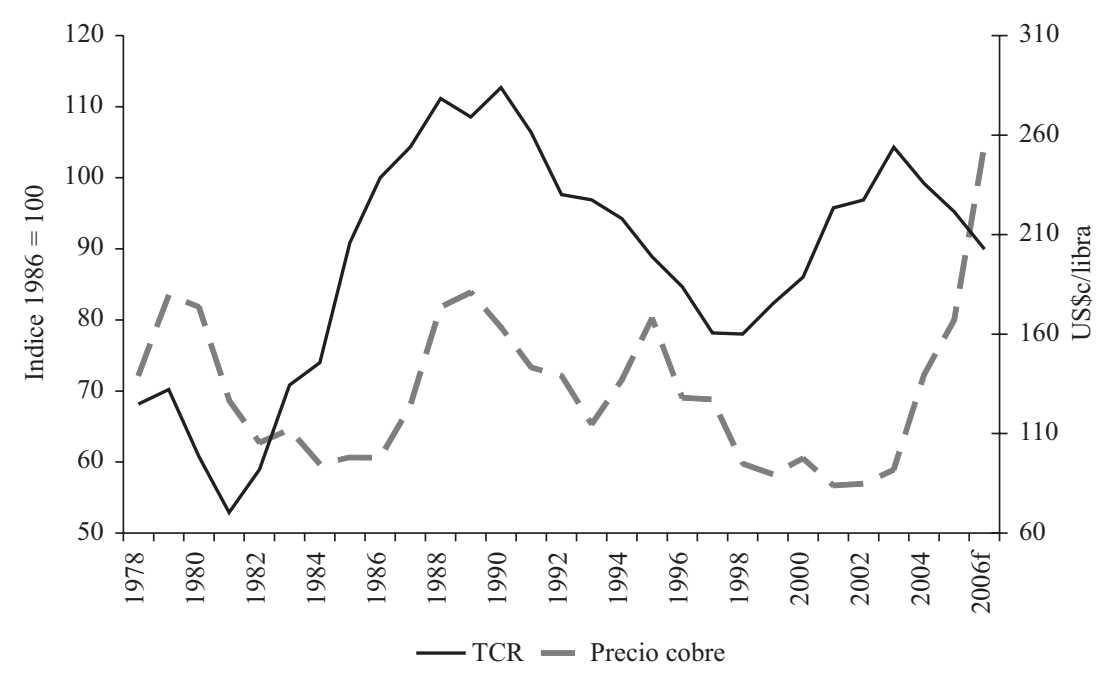

Fuente: Comisión Chilena del Cobre, Banco Central de Chile.

${ }^{11}$ La serie de TCR utilizada es la publicada por el Banco Central de Chile, la que fue revisada a partir del 2002 de acuerdo a Caputo y Dominichetti (2005). De aplicarse esta revisión hacia atrás tendría sólo efectos perceptibles a finales de los años 80 . 
una banda, y en 1997 se acumularon reservas internacionales equivalentes al 4\% del PIB, para tratar de frenar los efectos de una entrada de capitales que alcanzó al 8\% del PIB. La defensa del tipo de cambio estimuló la entrada de capitales. En esa época las expectativas de mercado eran una apreciación gradual, incluso eso estaba contemplado en el centro de la banda que contemplaba una caída real de $2 \%$. De esa forma, resultaba muy ventajoso endeudarse en el exterior.

La situación hoy es muy distinta. Durante esa época la apuesta cambiaria era unilateral. Todas apostaban a la apreciación. Hoy día el escenario es más balanceado, e incluso las expectativas tienden a ser estabilizadoras. En la actualidad, la encuesta de expectativas del Banco Central contempla una depreciación de 3\% a un año y 7\% a dos años. En los momentos de máxima depreciación a principios del 2003 se esperaba una caída de 3\% a 12 y 24 meses. Lo esperable es que haya igual predicción al alza que a la baja, y en nuestro caso se observa cierta reversión de los movimientos bruscos. El problema es que cuando el tipo de cambio se aprecia y se sigue esperando una apreciación, la situación se hace mucho más compleja de manejar, lo mismo ocurre en la dirección contraria.

Por lo tanto, hoy día tenemos un régimen de políticas macroeconómicas constituido por una regla fiscal contracíclica y un esquema de metas de inflación con tipo de cambio flexible que permiten explicar el grueso de la mayor estabilidad económica que hoy estamos observando ${ }^{12}$.

De esta forma podemos concluir que la economía chilena es hoy más estable, lo que tiene el costo aparente de limitar la expansión, pero el enorme beneficio de amortiguar las desaceleraciones.

\subsection{Algunas clarificaciones}

Mi discusión ha estado centrada en la estabilidad del PIB en torno a su tendencia de largo plazo. Por lo tanto, aquí no me refiero al crecimiento potencial de largo plazo de la economía. Es decir, a cuánto podemos crecer, dadas nuestras características económicas, nuestras políticas, instituciones y la calidad de nuestros recursos. Más allá de una posible desaceleración producto de la convergencia a niveles de PIB más elevados, creo que toda-

${ }^{12}$ El que las políticas macroeconómicas han hecho nuestra economía más estable ha sido recientemente confirmado en estudios de Larraín y Parro (2006), García y Restrepo (2006) y Medina y Soto (2006). Dichos trabajos enfatizan el rol de la actual regla fiscal versus no tener ninguna regla, y no hay evidencia comparando la regla actual con la que prevaleció en los noventa, lo que limita el uso de dichos resultados para comparaciones entre el régimen fiscal de los 90 y el actual. 
vía podemos seguir pensando que este crecimiento es cercano al 5\% por año, una cifra bastante alta para estándares internacionales. Más aún, es presumible que los efectos de convergencia hayan sido compensados por otros importantes avances ocurridos en la última década, como son el aumento de nuestros grados de apertura e integración a la economía global y la consolidación de la estabilidad macroeconómica ${ }^{13}$. Ciertamente con mejoras en aspectos fundamentales como la educación, el funcionamiento de los mercados y la innovación podríamos crecer más en el largo plazo, aunque obviamente son procesos que toman tiempo en rendir sus frutos y cuyo análisis está fuera del alcance del tema que nos ocupa hoy.

He sido explícito en indicar que con las políticas macroeconómicas hemos podido estabilizar el ciclo, sin embargo aún queda sin respuesta por qué tal vez el crecimiento este año no está más cerca, por ejemplo, de 7\%. No creo que haya una respuesta simple, pues la naturaleza del ciclo ha cambiado y lo que sabemos de nuestro crecimiento potencial también está sujeto a incertidumbre.

En segundo lugar, hay quienes dirán que hoy estamos sufriendo de la enfermedad holandesa. No existe evidencia para sostener esto. De hecho, las predicciones de la literatura de la enfermedad holandesa apuntan a que el crecimiento se daría en el sector de recursos naturales, a costa de una caída en la industria y otros sectores de bienes transables. Eso no ha ocurrido en Chile, salvo una desaceleración en la agricultura debido a factores puntuales de tipo climático y transición de cultivos tradicionales. Asimismo, la minería no muestra el dinamismo que se esperaría de un fenómeno de este tipo, y la economía sigue creciendo a tasas superiores al crecimiento del producto de tendencia, lo que ha permitido ir cerrando la brecha de producto. La economía y las exportaciones siguen creciendo, en particular las del sector industrial. Ciertamente hay una disminución en la rentabilidad del sector de bienes transables como resultado de la apreciación del peso, lo que no significa que esto sea una enfermedad. Esto es parte del proceso de ajuste a un aumento de los términos de intercambio.

También se podría pensar que el sector privado tiene una conducta sicológicamente bipolar: excesivamente eufórico al alza, excesivamente depresivo a la baja. La reciente evolución de la economía chilena muestra que

${ }^{13}$ En todo caso, el efecto más importante en los trabajos empíricos es el menor crecimiento como resultado de la convergencia, puesto que otros aspectos más cualitativos de los determinantes del crecimiento son difíciles de incluir en las proyecciones. Por esta razón, por ejemplo, en De Gregorio (2005) se presentan estimaciones de crecimiento de largo plazo que han caído unas décimas en el tiempo, aunque las diferencias no son estadísticamente significativas y se deben fundamentalmente a la convergencia y peores condiciones externas en la segunda mitad de la década pasada. 
éste no es el caso. No creo que podamos aludir a conductas perversas y desestabilizadoras del sector privado si no consideramos las distorsiones que induce la política económica. A mi juicio es la política económica la que induce comportamientos excesivamente gastadores o austeros. En todas las experiencias de desborde del gasto, aparentemente privado, ha sido la política macro la que lo ha estimulado. Por otra parte, la evidencia muestra que en Chile durante los noventa el componente del gasto que más aumentó fue la inversión y parte del estímulo también ocurrió por la mayor entrada de capitales, flujo que hoy no se observa (Spilimbergo, 2002) ${ }^{14}$. En este aspecto es necesario destacar que tal vez otro efecto estabilizador del ciclo tiene que ver con una característica del sector cobre: a diferencia de los noventa, hoy día no hay en carpeta un volumen de proyectos de inversión como aquellos que existieron en la década pasada.

Por último, es necesario destacar que la caída de la volatilidad del crecimiento ha ocurrido en muchas partes del mundo y no es sólo una característica de la economía chilena. En las Figuras No 7 y 8 se muestra para un grupo de países, así como para algunas agrupaciones, la máxima diferencia, positiva en la Figura $N^{0} 7$ y negativa en la 8, del crecimiento anual respecto del promedio para los años noventa y para la década actual ${ }^{15}$. En las figuras se muestra que efectivamente la volatilidad ha caído en muchos países, aunque esto no ha sucedido en América Latina como un todo ni tampoco en el mundo ni las economías avanzadas, sólo se ve una caída en la volatilidad del área Euro ${ }^{16}$. Lo que la mayoría de estos países comparten es que han adoptado metas de inflación y cuentan con altos grados de flexibilidad en el ámbito cambiario. Estos esquemas de metas de inflación, han sido mayoritariamente adoptados durante los noventa. Es decir, una mirada global a otros países sugiere que el marco de la política monetaria hace una diferencia en términos de volatilidad del producto ${ }^{17}$.

${ }^{14}$ En la actualidad el déficit privado en cuenta corriente es muy elevado, pues el superávit público es de varios puntos del PIB. Sin embargo, el moderado aumento de la demanda agregada indicaría que este déficit es el resultado de un lento crecimiento del ingreso privado nacional más que un aumento insostenible del gasto.

${ }^{15}$ Para el año 2006 se considera la proyección del World Economic Outlook del FMI.

${ }^{16}$ En el caso de América Latina la volatilidad medida comparando desviaciones mínimas y máximas respecto de la media sólo aumenta en Argentina, Uruguay y Venezuela.

${ }^{17}$ Mishkin y Schmidt-Hebbel (2005) muestran evidencia que apoya la idea de que los países que tienen metas de inflación tienen mejor desempeño macroeconómico. En particular, ellos argumentan que: "Output volatility has not worsened, and if anything improved, after the adoption of inflation targeting”. 
FIGURA N $N^{\circ}$ 7: VOLATILIDAD DEL PRODUCTO, EXPANSIONES

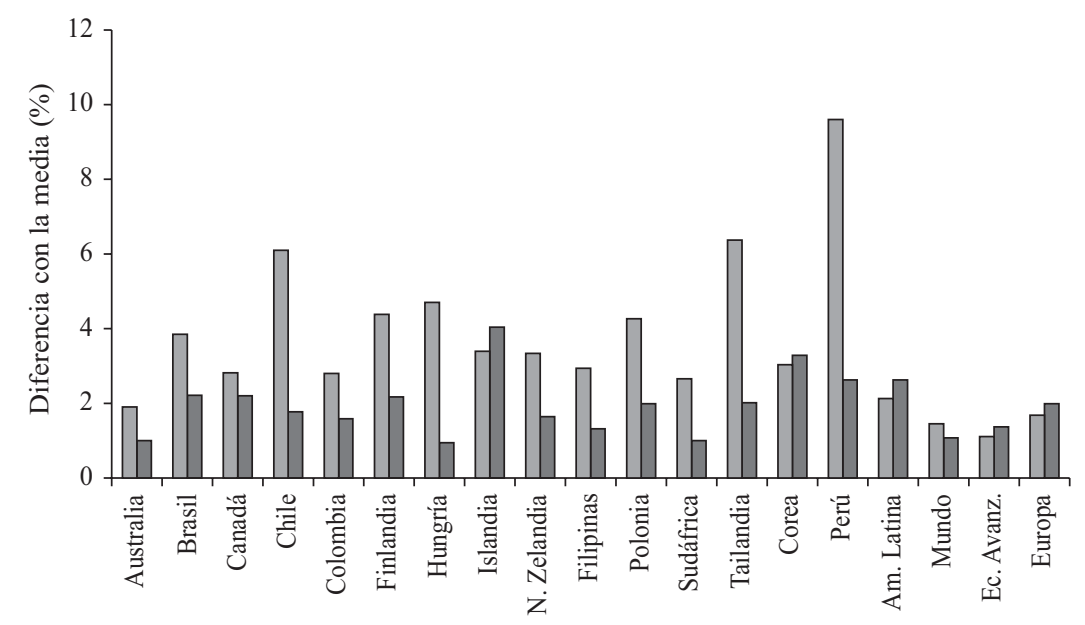

$\square 1990-1999 \quad \square 2000-2006$

FIGURA N ${ }^{\circ}$ 8: $\quad$ VOLATILIDAD DEL PRODUCTO, CONTRACCIONES

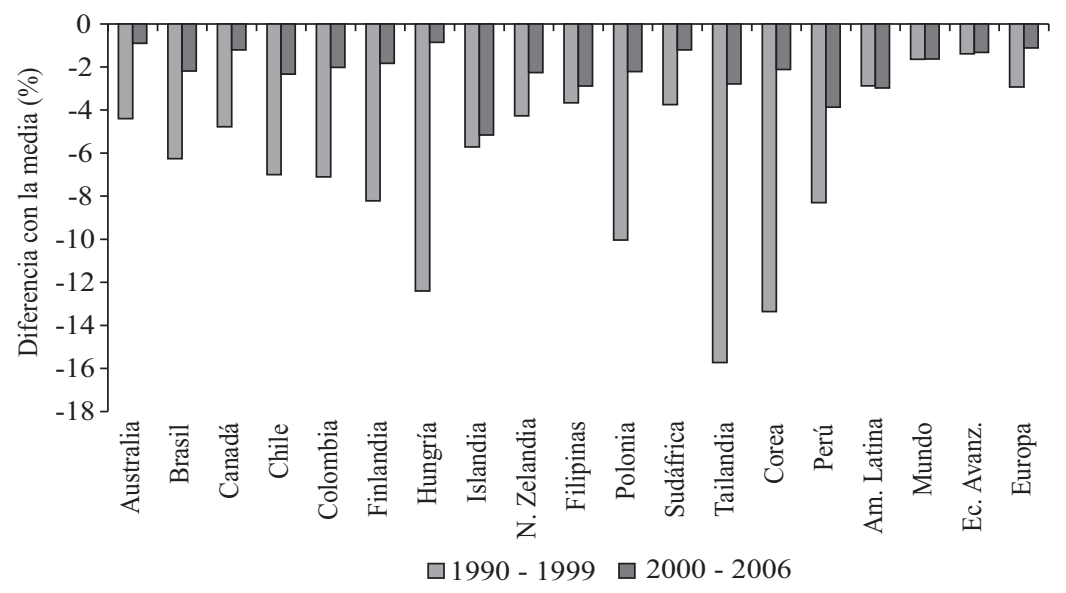




\section{2. ¿Qué hacer, qué no hacer y qué esperar?}

El aumento del precio del cobre nos ha hecho más ricos. Probablemente no de manera permanente, pero sí hemos tenido una ganancia que debemos manejar adecuadamente. Mientras más duradera sea esta ganancia, más beneficiosa será la bonanza y más desafíos tendremos para administrarla. Esa ganancia se debe gastar, pero el desafío más importante de la política fiscal es cómo graduar dicho gasto, dada la incertidumbre sobre su magnitud y las limitaciones que tiene la economía para absorberlo adecuadamente.

En consecuencia, no podemos gastar en el corto plazo todo el ingreso adicional, ni tampoco cargarlo a una rebaja de impuestos. De hecho todo lo que diga a continuación es, en una primera aproximación, válido tanto para un aumento del gasto como para una reducción de impuestos ${ }^{18}$. Una razón es la prudencia y consiste en ahorrar para la época en que los precios bajen. Un manejo razonable de las finanzas públicas sugiere ahorrar estos ingresos extraordinarios. Eso es exactamente lo que hace la regla del superávit estructural. Pero también hay razones de índole macro de por qué uno no puede gastar todo este ingreso, aunque sea duradero, y de ahí la necesidad de la gradualidad.

Pensemos como si Chile se hubiera ganado la lotería, pero el premio se entrega en anualidades y en algún momento se terminará sin aviso. Obviamente hay que ir ahorrando para cuando el premio se discontinúe. Esto es lo mismo que haría una familia si gana un premio de esta índole. Pero la familia también puede hacer grandes gastos que nunca antes pudo hacer, e incluso mantenerlos, porque tiene más riqueza. Podría comprar una casa, un auto, incluso una segunda casa si el premio es muy grande. Muchas de estas compras se pueden considerar inversión del hogar pues son bienes que prestarán servicios por muchos años, incluso cuando el premio se haya terminado. Todos estos gastos no tienen repercusiones agregadas. Sin embargo, esto no es así cuando un país se gana esta lotería, pues los gastos que realiza sí tienen repercusiones sobre toda la economía. Por lo tanto, la necesidad de cautela va más allá de la prudencia por la duración de este premio, va también por el impacto y la capacidad que tiene la economía de absorber este gasto.

\footnotetext{
${ }^{18}$ Dependiendo de en qué se gaste, o la naturaleza de una rebaja de impuestos, puede haber algún efecto diferente de más gasto o menos impuestos, pero a priori no hay ninguna razón de peso para pensar que sean muy distintos, en particular porque por lo general los aumentos de gasto se refieren a transferencias. Ahora bien, la combinación impuesto-gasto también tiene implicancias sobre el crecimiento de largo plazo.
} 
Lo que ocurre es que las economías tienen cierta capacidad máxima de producción en el corto plazo, e incluso también hay límites en plazos mayores. Si gastáramos todos estos ingresos adicionales no habría muchos más bienes que comprar, salvo que los importemos todos. Los precios de los bienes subirían, generando inflación. Esta presión sobre los precios, en particular de los bienes no transables cuyos precios no están sujetos a condiciones internacionales, generaría una apreciación del tipo de cambio real. Es decir, los bienes nacionales se encarecerían respecto de los bienes internacionales. Las tasas de interés subirían por la caída del ahorro y las presiones inflacionarias. De esta manera, el mayor gasto no se traduciría en mayor producción, sino que en mayor déficit de la cuenta corriente, mayores tasas de interés y una caída del tipo de cambio real.

En el caso extremo de que la producción es completamente rígida, lo que no es el caso pero sirve para aclarar el punto, un aumento del gasto de gobierno debe ser compensado con una caída en otros componentes del gasto y ellos son la inversión y las exportaciones netas vía un alza en las tasas de interés y una caída del tipo de cambio real. Es decir, los precios deben cambiar para abrir espacio al mayor gasto público. Más en general, efectivamente habrá presiones de demanda que aumentarán la producción en el corto plazo, pero esto no es duradero en la medida que lo que cambia no es la capacidad productiva y los precios relativos se alejan más de su equilibrio de largo plazo.

Se puede argumentar que es un pequeño precio a pagar por tener más gasto. Pero eso no es así. Cuando la economía está creciendo, hay presiones inflacionarias subyacentes producto de la mayor actividad, un ciclo de alzas en las tasas de interés y presiones a la apreciación del tipo de cambio, entonces no resulta conveniente agregarle impulso a la economía. Precisamente en eso consiste una política macroeconómica contracíclica, que atempere las fluctuaciones del producto y de los precios relativos. Por supuesto esto depende de grados y serán las autoridades fiscales quienes en definitiva decidirán la graduación de este proceso, pero es importante señalar los desafíos que tienen y las formas de abordarlo.

La regla del superávit estructural es un gran aporte a la estabilización del ciclo y constituye un gran avance en el manejo fiscal. Durante los noventa se dio un primer paso al acotar el crecimiento del gasto con el del PIB. Sin embargo, eso sí tenía un componente procíclico no deseable y que hubo que corregir con la recesión del 99. La regla actual asegura que si el producto está debajo del pleno empleo se pueda tener un déficit, y lo contrario ocurre en momentos de estrechez de capacidad. Esto ayuda a la estabilización del ciclo económico. 
Sin embargo, aún podría quedar un elemento procíclico que por primera vez se debería hacer evidente este año y que requiere de un examen cuidadoso. Éste se refiere al precio del cobre de largo plazo. Cuando el precio del cobre sube, es razonable suponer que también lo hace el precio de largo plazo que se usa en la regla. Esto ocurre debido a que la definición del precio de largo plazo del cobre de la regla es el correspondiente a los próximos diez años. Como los aumentos del precio del cobre tienen persistencia, debe también aumentar el promedio de los próximos diez años. Esto a su vez permite una mayor expansión fiscal. Ésta ocurre exactamente en momentos en que la economía no necesita de impulsos fiscales adicionales pues el PIB está por sobre su tendencia. Esto ocurre precisamente por la correlación positiva entre PIB, precio del cobre y actividad mundial. El caso opuesto sucedió cuando se inauguró la regla en un ciclo de precios del cobre muy bajos. En esa ocasión el precio era cercano a los 80 centavos de dólar la libra. De haber predicho el boom que experimentamos hoy, algo obviamente hipotético e imposible de concretar, la política fiscal hubiera sido más expansiva. Similarmente si mañana el precio inesperadamente colapsa, podremos haber hecho un presupuesto muy optimista.

Para ilustrar este punto se puede realizar el siguiente ejercicio. Supongamos que el precio del cobre es igual al supuesto en el último Informe de Política Monetaria, es decir este año sería en promedio 2,6 dólares por libra y el próximo año 2,25 dólares por libra. Supongamos ahora que se estima el precio del cobre de referencia, para los próximos diez años, usando un proceso autorregresivo de primer orden estimado con datos históricos. Esto es, se asume que los aumentos del precio del cobre persisten en el tiempo para ajustarse gradualmente a su tendencia de largo plazo que se asume en 1 dólar la libra. Finalmente, se usa un coeficiente de autocorrelación anual de 0,75 , se estima además que 10 centavos de dólar la libra representan mayores ingresos por $0,5 \%$ del PIB y se asume un crecimiento de tendencia del gasto de $5 \%{ }^{19}$. Estos cálculos son ilustrativos y usan estimaciones gruesas pero sirven para ilustrar el dilema de la política fiscal. Basado en estos parámetros se podría estimar cuál es la expansión del gasto público consistente con la regla. Al realizar este ejercicio se concluye que el gasto tendría una tasa de crecimiento anual de 15\% el 2007 y de 2\% el 2008. Claramente estas magnitudes exacerbarían el ciclo económico ${ }^{20}$.

\footnotetext{
${ }^{19}$ Habría también que agregar los intereses del superávit que se va acumulando, pero no debería haber un cambio muy significativo. Por ejemplo, un superávit de $5 \%$ del PIB en un año invertido a un $5 \%$ anual da $0,25 \%$ del PIB en intereses, lo que es aproximadamente un punto porcentual del gasto de gobierno.

${ }^{20}$ Esto asume que el resto de operación de la regla sigue su tendencia, lo que no necesariamente debiera ocurrir, pero las cifras que aquí se discuten son ilustrativas de las diferencias entre distintos escenarios.
} 
Alguien podría pensar que el comité de expertos debería considerar las repercusiones macro de su estimación y actuar con cautela. Pero ésta no es la idea del comité de expertos. A mi juicio las instituciones funcionan cuando hacen la tarea para la que fueron creadas. El desafío es entonces de la política fiscal y requiere de algunas decisiones importantes. Creo que podemos pensar en tres alternativas:

1. No hacer nada y dejar que ocurra dicha expansión del gasto y frenar posteriormente. La política fiscal es un dato para la política monetaria y presumiblemente esta expansión, en la medida que agregue presiones inflacionarias, significará tasas de interés más altas, tipo de cambio real más apreciado y más crecimiento el 2007. La situación se revertiría el 2008, con menor crecimiento, depreciación del tipo de cambio y tasas probablemente algo menores.

2. Ajustar el precio de largo plazo para darle mayor gradualidad al ajuste. Esto no necesariamente significa cambiar la regla, pero sí implica modificar su forma de implementación. Una opción es decir que se pensará en el precio de referencia mirando a 30 años hacia adelante, o alternativamente usar el precio de los próximos 10 años ponderado con el precio de referencia del año anterior. Esto último es igual a ajustarse gradualmente al precio de referencia.

3. Usar parte del potencial "gasto excesivo", aquel que pueda tener efectos indeseados sobre el ciclo, en áreas de poco impacto macro. Es decir que sean plenamente consistentes con la regla fiscal en su dimensión de ahorrar para cuando la bonanza haya pasado, y al mismo tiempo, ser consistentes con la capacidad de la economía de absorber el mayor gasto sin sobresaltos significativos. Aquí quisiera destacar y profundizar en una propuesta presentada en el mensaje presidencial del 21 de mayo que tiene que ver con el seguro de desempleo.

Si se quiere conciliar estabilidad macroeconómica con compromiso de gastar el componente permanente de la bonanza y, al mismo tiempo, hacer un importante esfuerzo en el ámbito social donde tenemos enormes desafíos, es posible incrementar el fondo solidario así como otorgar una transferencia de gasto a las cuentas individuales de seguro de desempleo. Esto es transformar ahorro público en ahorro privado ${ }^{21}$, lo que eventual-

${ }^{21}$ Esto es obvio en el caso de transferir recursos a las cuentas individuales, y en el caso del incremento del fondo solidario es más bien un tema de convenciones contables, aunque conceptualmente es ahorro del sector privado donde el beneficiario no está definido ex ante. 
mente no debiera tener impacto agregado. Tiene además otra gran contribución macroeconómica y es la de proveer un estabilizador automático al ciclo. Si hay algo que la política fiscal en Chile no ha tenido son estabilizadores automáticos fiscales por el lado del gasto. En países desarrollados estos son los generosos sistemas de subsidio de desempleo, los que generan expansiones automáticas en recesiones. Sin embargo, esto tiene serios problemas de incentivos y en Chile se innovó con un creativo sistema de seguro de desempleo basado en la capitalización individual y un fondo solidario. Esto puede tener un impacto estabilizador importante. Por lo tanto, a mi juicio, el incrementar el fondo solidario del seguro de desempleo así como entregar una transferencia a las cuentas individuales es macroeconómicamente positivo, es consistente con la regla fiscal y es de un importante contenido social pues entrega recursos a quienes más lo necesitan en tiempos de dificultad económica. En la medida que haya recursos en las cuentas de seguro de desempleo y no se ocupan, serán complementarios a las pensiones de los beneficiarios con el subsidio.

Aquí he esbozado una idea general que ciertamente requiere de análisis más detallado y cuidadoso. ¿Cómo asegurar y diseñar su progresividad en términos sociales? ¿Cómo diseñarlo de manera que no afecte negativamente las decisiones de trabajo y búsqueda de empleo? ¿Cómo graduarlo de manera que no se transforme en gasto corriente y sea efectivamente ahorro para situaciones de desempleo ${ }^{22}$ Ésas son algunas de las preguntas que se deberían responder para evaluar con más cuidado esta propuesta, pero puede contribuir a abordar los desafíos que enfrentará la política fiscal ${ }^{23}$.

${ }^{22}$ Cowan, Huneeus y Micco (2006) desarrollan una idea similar respecto de las cuentas individuales. Dicha propuesta consiste en suplementar con fondos públicos, con igual monto, las cotizaciones previsionales y al seguro de desempleo de las rentas más bajas.

${ }^{23}$ Post scriptum al 31 de agosto de 2006: Este trabajo fue preparado antes que se reunieran los comités de expertos que definen el producto potencial y el precio del cobre de largo plazo relevantes para la aplicación de la regla fiscal. El comité de expertos del precio del cobre determinó un precio de largo plazo de 121 centavos de dólar la libra. En este caso, y dadas declaraciones públicas del Ministerio de Hacienda, el crecimiento del gasto consistente con la regla fiscal y con el que se preparará el presupuesto para el 2007 será entre 8 y 10\%. La discusión del texto ha supuesto que el precio del cobre de largo plazo hubiese resultado más alto, llevando el crecimiento del gasto consistente con la regla a niveles de 15\%. Esta cifra ciertamente hubiera provocado serios trastornos macroeconómicos por la capacidad de absorción de la economía de un gasto de esas proporciones. Sin embargo, la discusión y propuesta discutida en este trabajo seguirá siendo válida en la medida que el elevado precio del cobre persista, y también tiene un mérito por sí misma en caso que se quieran discutir alternativas de ahorro de estos recursos, pues en este caso es transferir ahorro público a ahorro privado. Desde el punto de vista de la estabilidad macroeconómica, del funcionamiento del mercado del trabajo, y de apoyo a los sectores más necesitados, el fortalecimiento del seguro de desempleo es una de las áreas de mayor impacto. 
Otro aspecto importante a la hora de analizar la bonanza cuprífera es considerar sus implicancias cambiarias. Como ya discutí, la apreciación del tipo de cambio real es parte del ajuste al shock externo, y con un sistema de flotación así como con una política fiscal austera estos efectos son aminorados. Sin embargo mi impresión es que en algunos momentos se ha tendido a sobredimensionar el impacto que tiene sobre la economía. El sector productor de cobre es importante, pero una parte importante es participación externa y la parte nacional ahorra la mayoría de los ingresos extraordinarios.

La evidencia empírica, tanto para Chile como para países de la $\mathrm{OECD}^{23}$, indica que un aumento de los términos de intercambios de $10 \%$ resulta en una apreciación de alrededor de 3\%. Desde el 2003, cuando el tipo de cambio real alcanzó el máximo de esta década (Figura № 9), hasta el 2006, asumiendo que permanece constante por el resto del año, el tipo de cambio real se habría apreciado un $14 \%$. Por su parte aunque el precio real del cobre habría aumentado un 177\%, los términos de intercambio lo habrán hecho sólo un 49\%. Usando la elasticidad histórica podríamos explicar la

FIGURA N N $^{\circ}$ TIPO DE CAMBIO REAL A PESOS POR DÓLAR Y PRECIOS ACTUALES (Índice, base abril $2006=517,3$ )

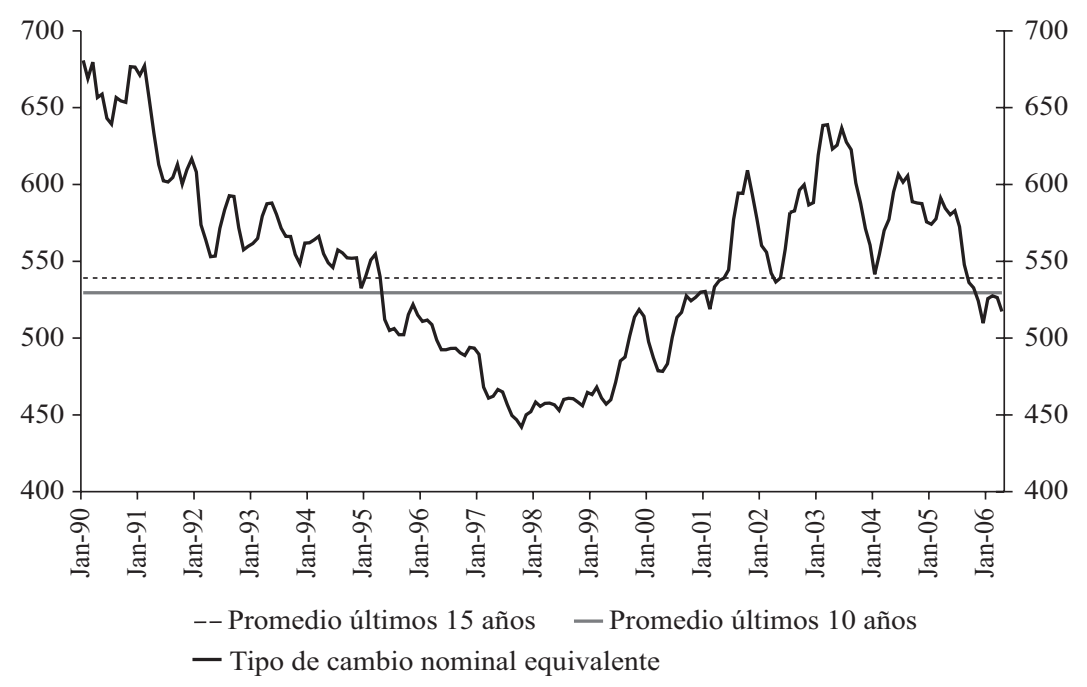

Fuente: Banco Central de Chile.

${ }^{23}$ Broda (2001), Caputo y Dominichetti (2005) y De Gregorio y Wolf (1994). 
caída ocurrida en el tipo de cambio real en los últimos tres años por el efecto de la ganancia de términos de intercambio. A esto debemos agregar el hecho de que la mayoría del aumento de ingresos proveniente del mejoramiento de las condiciones externas se ahorra y asimismo no existen presiones financieras, por ejemplo debido a la abundancia de ingreso de capitales, que presionen el tipo de cambio. Existen muchos otros factores que determinan el tipo de cambio, en particular en sus movimientos de corto plazo, pero estos cálculos sugieren que es difícil que en la coyuntura actual el tipo de cambio real se aprecie significativamente.

En materia monetaria-cambiaria hay que persistir con lo hecho hasta ahora. Como he tratado de argumentar, el esquema de política monetaria es el más adecuado en la actual coyuntura. De haber tenido este esquema a fines de los noventa habríamos pasado mucho mejor la crisis de los mercados emergentes. La política monetaria no se mide por el activismo de las autoridades ni por el número de medidas que se toman sino que por sus resultados. Lo que hemos visto en años recientes es la mejor contribución que las políticas macroeconómicas pueden hacer al crecimiento de largo plazo. Por supuesto, esto es una condición necesaria, no suficiente para crecer más.

La intervención sólo se justifica cuando se observa un desalineamiento significativo que ponga en peligro la estabilidad de la economía. Nunca se puede descartar, pero la experiencia muestra que pensar que se puede graduar una apreciación puede generar efectos indeseados a los que se quiere evitar. Hay que fortalecer la credibilidad de la flotación y las eventuales intervenciones. Se podría intervenir, quedar tranquilos y ser felicitados por pragmáticos, pero dañar seriamente la credibilidad y el funcionamiento futuro de nuestro esquema de política. Para intervenir necesitamos estar relativamente convencidos de que hay una sobrerreacción cambiaria, que ésta es dañina para la economía como un todo, y que la intervención tiene una probabilidad elevada de éxito.

Asimismo, para aumentar la efectividad y credibilidad del régimen cambiario debemos fortalecer este esquema a través de seguir mejorando la información acerca de las operaciones del banco central, incluyendo aquellas con el fisco, para evitar impactos indeseados en el corto plazo que puedan surgir del desconocimiento.

Por otra parte, hay que ser cuidadosos al argumentar que debemos arreglar problemas en sectores específicos por la vía de la intervención cambiaria. Bajo el supuesto de que es efectiva, sobre lo cual hay un debate no resuelto en particular cuando no se observan desalineamientos evidentes, se pide intervención cambiaria por el daño que sufren algunos sectores. 
Afectar el tipo de cambio para ir en ayuda de sectores específicos es una política muy ineficiente, porque el beneficio que reciben los productores afligidos también lo reciben todos los otros que no lo necesitan. Es decir, es una política muy mal focalizada.

Por último, quisiera referirme a otra propuesta que también circula en el ambiente y es la necesidad de aprovechar esta coyuntura para tomar un seguro macro para cuando caiga el precio del cobre y así evitar la excesiva volatilidad que nos generan los mercados financieros internacionales. Para discutir esta idea hay que distinguir entre el seguro que pueden tomar las empresas del que puede tomar el país. Las empresas pueden buscar mecanismos en los mercados de derivados para aprovechar el ciclo de precios altos. Incluso desde la política pública se pueden buscar seguros para problemas específicos, como el precio de los combustibles. Sin embargo, estos son problemas de las empresas o de políticas específicas, pero no son relevantes desde el punto de vista macro. Al respecto, como argumenté anteriormente, no hay evidencia de que en el pasado reciente hayamos sufrido shocks particularmente severos de los mercados financieros internacionales, de manera que comprar un seguro macro es algo de segundo orden, que además envuelve elevados riesgos al tratarse de instrumentos exóticos. En todo caso sí hay que aprovechar de seguir mejorando la integración financiera de nuestra economía y el funcionamiento del mercado de capitales chileno para proveer un mejor manejo de los riesgos de las empresas y facilitar el financiamiento de la inversión. No obstante, desde el punto de vista de comprar seguros, el mejor seguro para nuestra economía es una buena política macroeconómica, y creo que Chile ha caminado en la dirección correcta llegando a tener una política macroeconómica de primer nivel. El desafío permanente es saber administrarla.

\section{A modo de cierre}

Quisiera concluir señalando que soy un convencido de que el actual esquema de políticas macroeconómicas es el más eficiente para manejar esta bonanza. Como señalé anteriormente el éxito no se mide por el número de acciones ni la visibilidad de las medidas que se tomen. Tampoco hemos adoptado un régimen de moda ni es el resultado de una lección aprendida en los libros, u ortodoxia como dirán otros. Entiendo que a muchos les irrite que las autoridades digan que hay que hacer más de lo mismo, pero cuando estamos convencidos de que eso es lo mejor para el país debemos decirlo, así como también debemos ser capaces de ir aplicando las correcciones 
necesarias. Chile se ha caracterizado por su pragmatismo en materia de política económica y donde estamos hoy es el resultado de aprender de nuestros errores y también de los errores y aciertos de otros. No deberíamos temer a innovar ni cambiar el rumbo, pero no veo ninguna evidencia que nos sugiera innovar de manera importante.

\section{REFERENCIAS}

Bennett, H. y R. Valdés (2001): "Series de Términos de Intercambio de Frecuencia Mensual para la Economía Chilena: 1965-1999”. Documento de Trabajo N ${ }^{\circ}$ 98, Banco Central de Chile, mayo.

Bernanke, B., M. Gertler y S. Gilchrist (1996): "The Financial Accelerator and the Flight to Quality”. En The Review of Economic and Statistics, Vol. 78, No 1, pp. 1-15.

Broda, C. (2001): “Coping with Terms of Trade Shocks: Pegs versus Floats”. En American Economic Review, papers and proceedings, Vol. 1, No 92, pp. 376380.

Caballero, R. (2002): "Enfrentando la Vulnerabilidad Externa: Un Problema Financiero”. En Revista de Economía Chilena, Vol. 5, № 1, pp. 11-36.

Calvo, G. (2005): “Crises in Emerging Market Economies: A Global Perspective”. The Frank Graham Memorial Lecture, NBER Working Paper No 11305.

Caputo, R. y B. Dominichetti (2005): "Revisión Metodológica en el Cálculo del IPE e Implicancias sobre los Modelos de Tiempo para el TCR”. En Revista Economía Chilena, Vol. 8, $\mathrm{N}^{\circ}$ 1, Banco Central de Chile, abril.

Cowan, K. y J. De Gregorio (2005): "International Borrowing, Capital Controls and the Exchange Rate: The Chilean Experience”. Documento de Trabajo $\mathrm{N}^{\circ} 322$, Banco Central de Chile, por aparecer en Edwards, S. (ed.), Capital Controls and Capital Flows in Emerging Economies: Policies, Practices and Consequence, NBER, Chicago University Press.

Cowan, K., C. Huneeus y A. Micco (2006): "Una Propuesta para el Sistema de Pensiones: Un Subsidio a las Cotizaciones Individuales”. Mimeo enviado a la Comisión Reforma Provisional.

De Gregorio, J. (2005): “Crecimiento Económico en Chile: Evidencia, Fuentes y Perspectivas”. En Estudios Públicos No 98, otoño.

De Gregorio, J. y H. Wolf (1994): "Terms of Trade, Productivity and the Real Exchange Rate”. NBER Working Paper N ${ }^{\circ} 4807$.

Franken, H., G. Lefort y E. Parrado (2006): "Business Cycle Responses and Resilience of the Chilean Economy during the Last Fifty Years”. En R. Caballero, C. Calderón y L. Céspedes (eds.), External Vulnerability and Preventive Policies. Banco Central de Chile.

García, C. y J. Restrepo (2006): "The Case for a Countercyclical Rule-based Fiscal Regime”. Mimeo, Banco Central de Chile.

Larraín, F. y F. Parro (2006): “Chile Menos Volátil”. Mimeo, Universidad Católica.

Medina, L. y C. Soto (2006): “Copper Price, Fiscal Policy and Business Cycle in Chile”. Mimeo, Banco Central de Chile. 
Medina, J. y R. Valdés (1998): “Liquidez y Decisiones de Inversión en Chile: Evidencia de Sociedades Anónimas”. Documento de Trabajo No 25, Banco Central de Chile.

Mishkin, F. and K. Schmidt-Hebbel (2005): "Does Inflation Targeting Make a Difference?”. Por aparecer en K. Schmidt-Hebbel y F. Mishkin (eds.), Monetary Policy Under Inflation Targeting, Banco Central De Chile.

Spilimbergo, A. (2002): “Copper and the Chilean Economy 1960-1998”. En The Journal of Policy Reform, Vol. 5, № 2, pp. 115-126. 